Original Article

\title{
Revisiting The Value of Anti-streptolysin O Titer in Children with Rheumatic Fever
}

\author{
Mohamed S. Eid', Fatma Al Zahraa Mostafa1, Hend Hamed Tamim², Mohamed \\ Elberry ${ }^{1}$, Shaimaa Sayed ${ }^{*}$ * \\ 1 Pediatrics Department, Faculty of Medicine, Cairo University, Egypt; pediatric_medo@yahoo.com, \\ fatmaalzahraah@hotmail.com, mohammed_elberry@hotmail.com \\ 2 Clinical and Chemical Pathology Department, Faculty of Medicine, Cairo University, Egypt; hend- \\ tamim@hotmail.com \\ * Correspondence: shaimaasayed@kasralainy.edu.eg \\ Received: 15/11/2021; Accepted: 10/12/2021; Published online: 27/12/2021.
}

\begin{abstract}
:
Background: Acute rheumatic fever and rheumatic heart disease are the significant nonsuppurative late sequelae of group A beta hemolytic streptococcal infection. Anti-streptolysin $\mathrm{O}$ titer (ASOT) is a streptococcal antibody test that is used for the diagnosis of infections and is particularly useful in the diagnosis of acute rheumatic fever.

Aim of the work: To compare ASOT values in normal children to those with rheumatic fever and children with recurrent tonsillitis, and to evaluate its relationship with seasonal variations. Methods: A case-control study conducted on 300 children; 100 children with rheumatic fever, 100 with recurrent tonsillitis and 100 apparently healthy children as a control group. Children were subjected to history taking, echocardiography study and blood specimens for complete blood count, erythrocyte sedimentation rate, $\mathrm{C}$ reactive protein and ASOT were assessed using immunoturbidimetric assay tested by Cobas e501/502 (Roche). Human anti-streptolysin O antibodies (any type) agglutinate with latex particles coated with streptolysin $\mathrm{O}$ antigens.

Results: Mean and SD of age in each group was $10.2 \pm 3$ years for rheumatic fever group, $7.3 \pm$ 3.8 years for recurrent tonsillitis group and $5.4 \pm 2.4$ years for control group. Rheumatic fever group included 49 males and 51 females, recurrent tonsillitis group included 58 males and 42 females, control group included 63 males and 37 females. ASOT ranged between 261-860 (mean \pm $\mathrm{SD}=475.1 \pm 214.2), 210-813($ mean $\pm \mathrm{SD}=257.6 \pm 56.4)$ and $65-820($ mean $\pm \mathrm{SD}=158.8 \pm 105.4)$ among those with rheumatic fever, tonsillitis and control group respectively $(\mathrm{p}=0.001)$. ASOT level between 400-800 IU/ml were common among patients with rheumatic fever and levels between 200-400 IU/ml were common with recurrent tonsillitis $(\mathrm{p}<0.001)$ with lack of diagnostic cut-off.

Conclusion: ASOT levels are higher in rheumatic fever patients compared with recurrent tonsillitis and normal children. There is lack of diagnostic cut-off of ASOT, hence the value of ASOT is only within scoring system to avoid over or under diagnosis of acute rheumatic fever. An isolated high ASOT is not sufficient to diagnose or rule out acute rheumatic fever.

Level of Evidence of Study: IIA (1).

Keywords: Acute rheumatic fever; Anti-streptolysin O titer; Recurrent tonsillitis.

Abbreviations: ARF: rheumatic fever; ASOT: Anti-streptolysin O titer; RHD: rheumatic heart disease.
\end{abstract}

\section{Introduction}

Although acute rheumatic fever (ARF) is relatively rare in developed economies, it is much more common in the developing world (2). It has been estimated that worldwide 15.6 million people have rheumatic heart disease (RHD) (3). Many regions have this major public health problem as the Middle East, the Indian subcontinent, some areas of Africa and South America. The prevalence of RHD was reported to be 5.1 per 1000 in school children in Egypt (4). So, rheumatic fever is still one of Egypt's major public health problems (5). 
Evidence of a host immune response to group A beta hemolytic streptococcal throat infection is required to confirm the diagnosis of rheumatic fever $(3,4)$. Although anti-streptolysin $\mathrm{O}$ titer (ASOT) has been used to diagnose recent streptococcal infection, interpretative criteria suggested by manufacturers of commercial antibody test kits are not suitable for children. It is not uncommon for laboratory personnel and physicians to misinterpret streptococcal antibody titers because of a failure to appreciate that the normal levels of these antibodies are higher among school-age children than among adults (6). Again it is important to note that these kits react to all antibody classes IgG and IgM against streptococcal streptolysin. Hence, at any point the titer reflects a previous infection, but does not reflect if it is IgM recent infection, or IgG old infection (7). However, there are some factors that may be confusing and might hinder interpretation. School-age children develop streptococcal infections more frequently and may present higher ASOT values due to infections that are coincident with but unrelated to diagnosis. Also, patients with rheumatic fever who have isolated manifestation of chorea may present with normal ASOT levels due to the latency of 1 to 6 months between infection and clinical manifestations (8). Upper limit of normal values of ASOT are known to vary also with respect to different geographical locations, season, and site of infection. Since over- diagnosis of ARF based on a raised ASOT is not uncommon, its upper limit of normal value in normal children is of importance to interpret ASOT in patients suspected to have ARF (9).

The aim of the current study is to compare ASOT values in normal children to those with rheumatic fever and children with recurrent tonsillitis, and to evaluate the variations of ASOT with tonsillectomy and with long acting penicillin compliance.

\section{Subjects and Methods}

This is a case control study performed on 300 children at Cairo University Children's Hospital. The current study has been approved by the Research Ethics Committee, Faculty of Medicine, Cairo University. The study conformed with the requirements of Revised Helsinki Declaration of Bioethics (2013) (10).

\section{Participants}

The study included 3 groups; 100 children with recurrent tonsillitis, 100 children with rheumatic fever and 100 apparently healthy children as a control group. The inclusion criteria were: children aged from $3-18$ years, from both sexes, children with rheumatic fever and RHD who had documented serial ASOT titers (rheumatic fever group), children with recurrent tonsillitis without tonsillectomy and who undergo tonsillectomy with available serial ASOT titers before and after tonsillectomy (recurrent tonsillitis group), apparently healthy children with no history of recent tonsillitis or sore throat (control group). The children will congenital heart disease were not included in the study.

\section{Methods}

Each child was subjected to history taking including age and sex. Confirmed cases of ARF needed clinical definitive evidence \& laboratory suggestive evidence of rheumatic fever according to Modified Jones criteria 2015 of American heart association of ARF and RHD (11). History of presence of arthritis, available oropharyngeal cultures, antibiotic treatment, echocardiography study were documented and blood specimens were analyzed at clinical and chemical department at Cairo university for complete blood count (on Sysmex xn 1000), erythrocyte sedimentation rate (ESR), C reactive protein (CRP) and ASOT (by ImmunoTurbidimetric method). The principle of ASOT testing is immunoturbidimetric assay tested by Cobas e501/502 (Roche). Human anti-streptolysin $\mathrm{O}$ antibodies (any type) agglutinate with latex particles coated with streptolysin $\mathrm{O}$ antigens. The participate was determined turbidimetrically.

\section{Statistical Analysis}

Data were statistically described in terms of mean \pm standard deviation $( \pm \mathrm{SD})$, median and range, or frequencies (number of cases) and percentages when appropriate. Comparison of numerical variables between the study groups was done using Student $t$ test for independent samples in comparing 2 groups and one way analysis of variance (ANOVA) test with posthoc 
multiple 2-group comparisons for comparing the 3 groups. For comparing categorical data, Chisquare $(\mathrm{x} 2)$ test was performed. Exact test was used instead when the expected frequency is less than 5. Correlation between various variables was done using Pearson correlation equation for linear relation of normally distributed variables and Spearman rank correlation equation for non-normal variables/non-linear monotonic relation. Accuracy was represented using the terms sensitivity, and specificity.

Receiver operator characteristic (ROC) analysis was used to determine the optimum cut off value for ASOT in differentiating rheumatic from control and tonsillitis groups. Two sided p-values less than 0.05 was considered statistically significant. All statistical calculations were done using computer program IBM SPSS (Statistical Package for the Social Science; IBM Corp, Armonk, NY, USA) release 22 for Microsoft Windows.

ROC Curve graphical plot that illustrates the diagnostic ability of a binary classifier system as its discrimination threshold is varied. It was created by plotting the true positive rate against the false positive rate at various threshold settings.

\section{Results}

\section{Clinical Features}

This was a case control study included 3 groups (100 children in each); patients with rheumatic fever, patients with recurrent tonsillitis and control groups. Mean and SD of age in each group was $10.2 \pm 3$ years for rheumatic fever group, $7.3 \pm 3.8$ years for recurrent tonsillitis group and 5.4 \pm 2.4 years for control group. Rheumatic fever group included 49 males and 51 females, recurrent tonsillitis group included 58 males and 42 females, control group included 63 males and 37 females. Regarding seasonal variation; 26 cases $(26 \%)$ of recurrent tonsillitis had seasonal variation, 23 cases were reported in winter. While in rheumatic group; 9 cases (9\%) only reported seasonal variation and the majority of cases showed non-seasonal variation. Regarding the presence of history of arthritis; 36 patients (36\%) of the rheumatic group had history of arthritis. While 3 patients of recurrent tonsillitis group (3\%) had history of arthritis and were followed up by serial echocardiography studies which were all free. There was statistically significant increase in the number of children suffered from arthritis between the 2 groups with $\mathrm{p}$ value $<0.001$.

\section{Laboratory Findings}

Laboratory findings are demonstrated in table 1. ESR and platelets counts in the rheumatic fever group were statistically significant higher than in the recurrent tonsillitis group. Most of studied rheumatic \& tonsillitis patients had negative CRP at the time of the study with a percent of $66 \% \& 76 \%$ respectively.

Table 1: Laboratory parameters of studied groups.

\begin{tabular}{|c|c|c|c|c|c|c|c|}
\hline & & & \multicolumn{2}{|c|}{ Rheumatic $(\mathrm{n}=100)$} & \multicolumn{2}{|c|}{ Tonsillitis $(n=100)$} & \multirow{2}{*}{ Pvalue } \\
\hline & & & \multicolumn{2}{|c|}{ Mean (SD) } & \multicolumn{2}{|c|}{ Mean (SD) } & \\
\hline ESR & & & \multicolumn{2}{|c|}{$29.9(21.9)$} & \multicolumn{2}{|c|}{$21.1(9.3)$} & $<0.001$ \\
\hline Hemoglobin & & & \multicolumn{2}{|c|}{$11.2(1.2)$} & \multicolumn{2}{|c|}{$11.1(1.0)$} & 0.428 \\
\hline Total leukocyte c & unt & & \multicolumn{2}{|c|}{$7.2(2.0)$} & \multicolumn{2}{|c|}{$7.7(1.4)$} & 0.033 \\
\hline \multirow{2}{*}{\multicolumn{3}{|c|}{ Platelets }} & \multicolumn{2}{|c|}{$325.8(88.3)$} & \multicolumn{2}{|c|}{$274.6(80.4)$} & $<0.001$ \\
\hline & & & \multicolumn{2}{|c|}{ Number } & \multicolumn{2}{|c|}{ Number } & \\
\hline \multirow{4}{*}{ Positive CRP ${ }^{\dagger}$} & & & \multirow{2}{*}{\multicolumn{2}{|c|}{$\begin{array}{l}34 \\
66\end{array}$}} & \multicolumn{2}{|c|}{24} & \multirow[t]{2}{*}{0.119} \\
\hline & & & & & & & \\
\hline & \multicolumn{2}{|c|}{ Controls $(n=100)$} & \multicolumn{2}{|c|}{ Rheumatic $(n=100)$} & \multicolumn{2}{|c|}{ Tonsillitis $(n=100)$} & \multirow{3}{*}{$<0.001$} \\
\hline & $\begin{array}{c}\text { Mean } \\
\text { (SD) }\end{array}$ & $\begin{array}{c}95 \% \\
\text { CI }\end{array}$ & $\begin{array}{c}\text { Mean } \\
\text { (SD) }\end{array}$ & $\begin{array}{l}95 \% \\
\text { CI }\end{array}$ & $\begin{array}{r}\text { Mean } \\
\text { (SD) }\end{array}$ & $\begin{array}{c}95 \% \\
\text { CI }\end{array}$ & \\
\hline \multirow[t]{2}{*}{ ASOT values } & $\begin{array}{c}158.8 \\
(105.4) \\
\end{array}$ & $\begin{array}{c}73.9, \\
136.9 \\
\end{array}$ & $\begin{array}{c}475.1 \\
(214.2) \\
\end{array}$ & $\begin{array}{l}432.6 \\
517.6\end{array}$ & $\begin{array}{l}257.6 \\
(56.4)\end{array}$ & $\begin{array}{l}246.4 \\
268.8\end{array}$ & \\
\hline & \multicolumn{2}{|c|}{ Number } & \multicolumn{2}{|c|}{ Number } & \multicolumn{2}{|c|}{ Number } & \\
\hline ASOT $<200$ & \multicolumn{2}{|l|}{82} & \multicolumn{2}{|c|}{3} & \multicolumn{2}{|c|}{11} & \multirow{4}{*}{$<0.001$} \\
\hline $200-400$ & \multirow{2}{*}{\multicolumn{2}{|c|}{$\frac{13}{5}$}} & \multicolumn{2}{|c|}{29} & \multicolumn{2}{|c|}{86} & \\
\hline $400-800$ & & & \multirow{2}{*}{\multicolumn{2}{|c|}{$\begin{array}{l}54 \\
14\end{array}$}} & & & \\
\hline $800-1200$ & \multicolumn{2}{|l|}{0} & & & & & \\
\hline
\end{tabular}

ASOT: anti-streptolysin O titer in IU/ml; CRP: C reactive protein; ESR: erythrocyte sedimentation rate; $\mathrm{P}$ value: probability value; SD: standard deviation 
Regarding ASOT, most of rheumatic fever group had their ASOT categories between 400 and $800 \mathrm{IU} / \mathrm{ml}$ (54\%), while most of tonsillitis group had their ASOT categories in between 200 and $400 \mathrm{IU} / \mathrm{ml}$ (86\%). On the other hand, most of the control group had their ASOT levels categories below $200 \mathrm{IU} / \mathrm{ml}(82 \%)$. ASOT values were significant high among rheumatic group (475.1 \pm $214.2 \mathrm{IU} / \mathrm{ml})$ compared to recurrent tonsillitis group $(257.6 \pm 56.4 \mathrm{IU} / \mathrm{ml}) \&$ the control group $(105.4 \pm 158.8 \mathrm{IU} / \mathrm{ml})$ as demonstrated in table 1 .

In the current study, children in each group were reclassified according to their age into 3 subgroups; either $\leq 5$ years, 6 - 10 years and those $>10$ years. There was no significant difference in ASOT levels between children of the control, rheumatic and tonsillitis groups and their different age groups.

Table 2: ASOT analysis within rheumatic fever and recurrent tonsillitis groups.

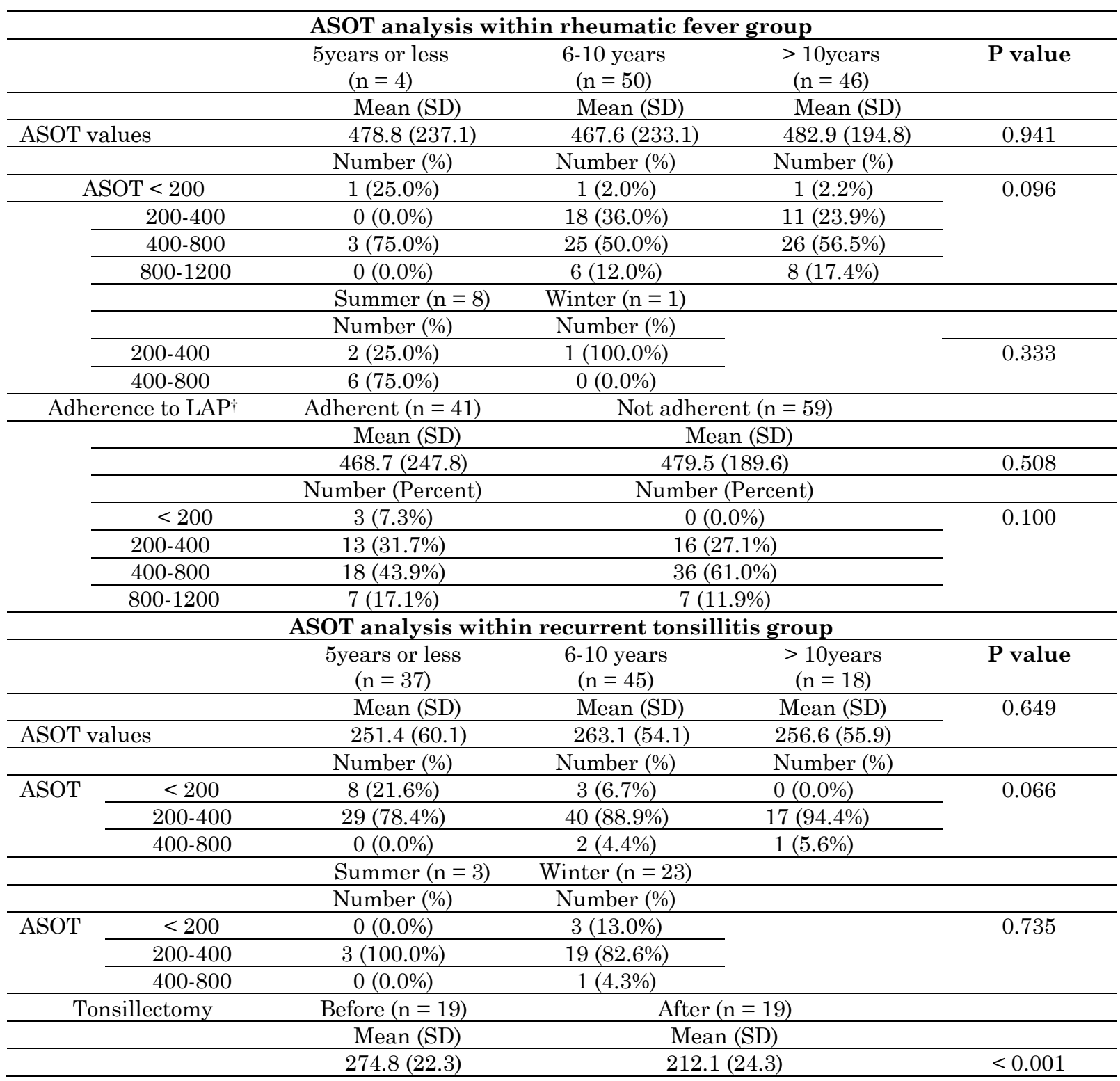

ASOT: anti-streptolysin O titer in IU/ml; LAP: long acting penicillin.

Associations and correlations of ASOT with clinicopathological features in the studied groups

In the rheumatic fever group, we did not find any statistically significant difference in ASOT levels and their age, compliance on long acting penicillin or seasonal variations as shown in table 2. In tonsillectomy group, we did not find any statistically significant difference in ASOT levels and their age or seasonal variations as shown in table 2 . 


\section{Receiver Operating Characteristic analysis for determination of the best cut- off limit of ASOT in discriminating rheumatic group from \\ Control group}

The best cut-off limit was at $398.5 \mathrm{IU} / \mathrm{ml}$ that achieved the lowest sensitivity of true positives $68 \%$ with highest specificity to detect true negative cases $95 \%$ that (the best clinical validity). Another cut-off limit at $97 \mathrm{IU} / \mathrm{ml}$ that achieve the highest sensitivity $100 \%$ with lowest specificity $69 \%$, that indicates the false negative patients as demonstrated at figure 1 . The choice of the 80th percentile cut-off rather than more traditional upper-limit-of-normal calculations is based upon studies that found that more than 80 to $90 \%$ of patients with ARF have streptococcal titers that are above the 80th percentile for the healthy controls with no clinical evidence of recent streptococcal infection (9). These cut-offs are not $100 \%$, hence, there are overlap in levels of ASOT between the 3 studied groups, and the ASOT cannot be used solely to diagnose any of the 3 groups.

Recurrent tonsillitis group

The best cut-off limit at was $390 \mathrm{IU} / \mathrm{ml}$ that achieved the lowest sensitivity $68 \%$ with highest specificity $97 \%$ which indicates the false positive patients. Another cut-off limit at was $249 \mathrm{IU} / \mathrm{ml}$ that achieved the highest sensitivity $100 \%$ with lowest specificity $69 \%$ indicating the false negative patients demonstrated at figure 2 .

ROC Curve

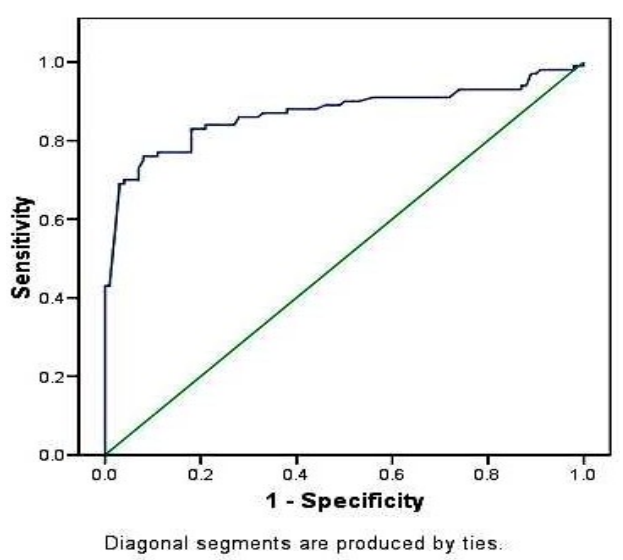

Figure 1: Anti streptolysin O titer in discriminating rheumatic fever group from control group.
Roc Curve

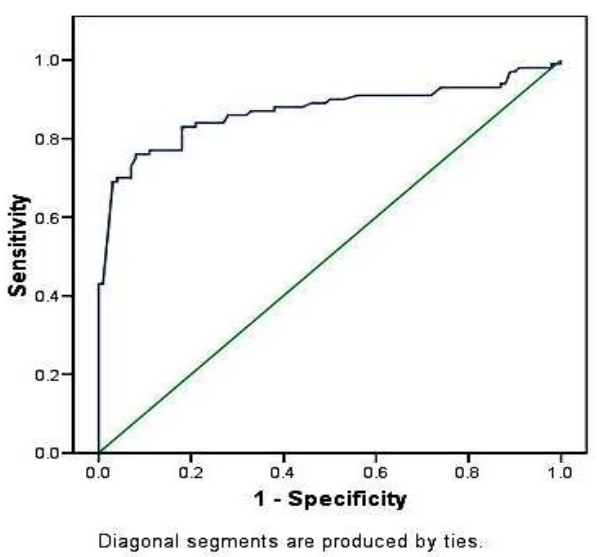

Figure 2: Anti streptolysin $\mathrm{O}$ titer in discriminating rheumatic fever group from recurrent tonsillitis group.

\section{Discussion}

Rheumatic fever is an autoimmune, multi-organ inflammatory disease that occurs as a result of infection with group A B-hemolytic streptococcal in genetically susceptible individuals. Acute rheumatic fever (ARF) and its sequel, rheumatic heart disease (RHD), cause significant morbidity and mortality in developing countries. They are under-recognized as global health problems (12). Rheumatic fever diagnosis relies upon a long renowned Johns criteria which is scoring system that includes clinical and lab findings. The criteria were revised by American Heart Association. All scoring criteria required evidence of antecedent group A B-hemolytic streptococcal infection (13). The widespread testing for elevated/increasing anti-streptococcal antibody titer in serum, has led to overdiagnosis of ARF based on raised ASOT (9) and subsequent over-management with all its psychological, social and financial burdens.

Ninety-six percent of our studied cohort with ARF were above 5 years of age. It was noticed that age between 5-15 years carries the highest risk for ARF in Egypt because at these ages children are in schools which are poorly ventilated and overcrowded and these favor the spread of streptococcal infection $(14,15)$. The need for proper aeration at school and sanitary housing seems to be the necessary corner stone in the prevention of ARF (16).

The levels of ASOT were not influenced by the age groups our studied cohorts. This is not congruent with others (9), as it seems that living in endemic areas with high rates of clinical/ subclinical infections (17) with streptococci produces a cumulative elevated titer. Also the 
recognition of presence of Streptococcus pneumoniae as a part of the nasopharyngeal microbiome (18) of children might explain the prevalence of the higher ASOT titer among the control group. Moreover, the commercially available kits detect both ASOT IgG and IgM, so the titer does not allow the interpreter to know if it is a recent or an old infection. Hence, if even if other studies have demonstrated a statistical difference according to age or could not (19), there is very little value of level of ASOT in diagnosis of ARF or ruling it out.

The noted seasonal variation in titer of ASOT which are most commonly observed in late winter and early spring is expected, but has no bearing on diagnosis of tonsillitis or otherwise (20). In the current study, in the recurrent tonsillitis group, ASOT significantly decreased after tonsillectomy than its value before it $(212.1 \leq \pm 24.3 \mathrm{IU} / \mathrm{ml}$ versus $274.8 \pm 22.3 \mathrm{IU} / \mathrm{ml}$ respectively, $\mathrm{p}$ value $<0.001)$. Other reports of conflicting and non-consistent results have been reported where children with recurrent acute tonsillitis who underwent tonsillectomy, had negative serologic results for ASOT after surgery or did not $(21,22)$. It seems that tonsillectomy reduces the rate of infection, or that the immune response to ASOT wanes away.

Other parameters were noted among the group with ARF. The mean value of ESR \& platelets were significantly higher in rheumatic fever group than recurrent tonsillitis group ( $p$ value $<0.001$ ). This may be due to increased rate of platelet production in bone marrow, and it may be used as an indicator of platelet activation and severity of inflammation, and underscores the value of searching for the criteria necessary to make a diagnosis of ARF (23).

ROC curve analysis was performed to evaluate the usefulness of ASOT among our studied 3 groups as a potential diagnostic marker to discriminate between the rheumatic fever group and the control. But even the best cut-off limit at $398.5 \mathrm{IU} / \mathrm{ml}$ that achieved the lowest sensitivity $68 \%$ with highest specificity $95 \%$ does not allow decision making as to rule out ARF or ascertain it. Same findings were reported by Saini and coworkers in 2019, where ROC curves of ASOT in $\mathrm{ARF}$ showed that a cutoff value more than $297 \mathrm{IU} / \mathrm{ml}$ has a sensitivity of $53.8 \%$ and specificity of $95 \%$ (24), and reported by Sanjeevaiah and coworkers in 2019, where the average cut-off ASOT value was $256.53 \mathrm{IU} / \mathrm{ml}$ with good specificity (95.0\%), sensitivity (88.05\%) (6). An extensive research was performed among the children and even normal values of ASOT in healthy children were not fixed and proposed to be either less than $239 \mathrm{IU} / \mathrm{ml}$ (25) or less than $250 \mathrm{IU} / \mathrm{ml}$, or even less than $305 \mathrm{IU} / \mathrm{ml}(26)$. Also, in the study conducted by Asfaw et al.2018, demonstrated that the average ASOT value upper limits of normal was $360 \mathrm{IU} / \mathrm{ml}$. This finding shows that ASOT upper limits of normal may be similar to those reported in countries with different climates and populations (8).

Hence, there is no consensus on an accurate diagnostic cut-off of ASOT value. This is of a great importance as over diagnosis of ARF based on a raised ASOT value in endemic areas is a common problem (9).

\section{Conclusions}

ASOT levels are higher in rheumatic fever patients compared with children with recurrent tonsillitis and normal children but an isolated high ASOT level is not sufficient to diagnose ARF. The lack of diagnostic cut-off of ASOT is of great importance; hence the value of ASOT is only valid within scoring system to avoid over diagnosis of ARF. ASOT levels in between 400-800 $\mathrm{IU} / \mathrm{ml}$ are common among children who suffer from rheumatic fever, but are detected among those with tonsillitis as well. Seasonal variation of ASOT levels is not that apparent finding. It is important to note that commercially available kits react to all antibody classes IgG and IgM against streptococcal streptolysin. Hence, at any point the titer reflects a previous infection, but does not reflect if it is IgM recent infection, or IgG old infection.

\section{Author Contributions:}

All authors shared in conceptualization, supervising, data curation, data analysis, writing original draft, data interpretation, writing original draft, supervising and revising. All authors reviewed the final manuscript. All authors have read and agreed to the published version of the manuscript.

\section{FUNDING}

Authors declare there was no extramural funding provided for this study. 


\section{CONFLICT OF INTEREST}

The authors declare no conflict of interest in connection with the study.

\section{References}

1. S. Tenny, M. Varacallo, Evidence Based Medicine. (StatPearls Publishing; Treasure Island (FL), 2020; https://www.ncbi.nlm.nih.gov/books/NBK470182/).

2. R. H. Webb, C. Grant, A. Harnden, Acute rheumatic fever. BMJ, h3443 (2015).

3. J. R. Carapetis, A. Beaton, M. W. Cunningham, L. Guilherme, G. Karthikeyan, B. M. Mayosi, C. Sable, A. Steer, N. Wilson, R. Wyber, L. Zühlke, Acute rheumatic fever and rheumatic heart disease. Nat. Rev. Dis. Primer. 2, 15084 (2016).

4. S. Kotit, K. Said, A. ElFaramawy, H. Mahmoud, D. I. W. Phillips, M. H. Yacoub, Prevalence and prognostic value of echocardiographic screening for rheumatic heart disease. Open Heart. 4, e000702 (2017).

5. S. Deraz, R. El-Zayat, A. Badawy, Prevalence of rheumatic heart disease in school children aged 6-15 years. Menoufia Med. J. 33, 162 (2020).

6. C. Reddy Parthihally Sanjeevaiah, Prediction of Upper Limits of Normal Values of Anti Streptolysin 'O' Titres in Normal Healthy School Going Children of 6-16 Years. Am. J. Pediatr. 5, 74 (2019).

7. S. Fujikawa, Y. Hanawa, H. Ito, M. Ohkuni, Y. Todome, H. Ohkuni, Streptococcal Antibody: As an Indicator of Tonsillectomy. Acta Otolaryngol. (Stockh.). 105, 286-291 (1988).

8. T. Asfaw, Antistreptolysin O Titers: Normal Values for Children Ages 5 to 15 at Debre Berhan Referral Hospital, Ethiopia. J. Fam. Med. Health Care. 4, 1 (2018).

9. A. A. Kotby, N. M. Habeeb, S. Ezz El Arab, Antistreptolysin O titer in health and disease: levels and significance. Pediatr. Rep. 4, 8 (2012).

10. World Medical Association, WMA Declaration of Helsinki- Ethical Principles for Medical Research Involving Human Subjects (2013), (available at https://www.wma.net/policiespost/wma-declaration-of-helsinki-ethical-principles-for-medical-research-involving-humansubjects/2013/).

11. M. H. Gewitz, R. S. Baltimore, L. Y. Tani, C. A. Sable, S. T. Shulman, J. Carapetis, B. Remenyi, K. A. Taubert, A. F. Bolger, L. Beerman, B. M. Mayosi, A. Beaton, N. G. Pandian, E. L. Kaplan, Revision of the Jones Criteria for the Diagnosis of Acute Rheumatic Fever in the Era of Doppler Echocardiography: A Scientific Statement From the American Heart Association. Circulation. 131, 1806-1818 (2015).

12. J. Alqanatish, A. Alfadhel, A. Albelali, D. Alqahtani, Acute rheumatic fever diagnosis and management: Review of the global implications of the new revised diagnostic criteria with a focus on Saudi Arabia. J. Saudi Heart Assoc. 31, 273-281 (2019).

13. I. Szczygielska, E. Hernik, B. Kołodziejczyk, A. Gazda, M. Maślińska, P. Gietka, Rheumatic fever - new diagnostic criteria. Reumatologia/Rheumatology. 56, 37-41 (2018).

14. A. Ghamrawy, N. N. Ibrahim, E. W. Abd El-Wahab, How accurate is the diagnosis of rheumatic fever in Egypt? Data from the national rheumatic heart disease prevention and control program (2006-2018). PLoS Negl. Trop. Dis. 14, e0008558 (2020).

15. Doaa Mohamed El-Amrousy, Hassan Al-Asy, Wegdan Mawlana, Acute Rheumatic Fever In Egyptian Children: A 30- Year Experience in a Tertiary Hospital, e220 (2014).

16. E. Okello, B. Kakande, E. Sebatta, J. Kayima, M. Kuteesa, B. Mutatina, W. Nyakoojo, P. Lwabi, C. K. Mondo, R. Odoi-Adome, F. Juergen, Socioeconomic and Environmental Risk Factors among Rheumatic Heart Disease Patients in Uganda. PLoS ONE. 7, e43917 (2012).

17. R. Gupta, K. Prakash, A. K. Kapoor, Subclinical group A streptococcal throat infection in school children. Indian Pediatr. 29, 1491-1494 (1992).

18. C. Basanti, M. Kotb, A. Sayed, M. Abdelfattah, M. Ghanem, A. Abdelmegeid, Nasopharyngeal Microbiome Composition is Different Among Children with Bronchial Asthma. Pediatr. Sci. J. 1, 77-88 (2021).

19. M. Danchin, J. Carlin, W. Devenish, T. Nolan, J. Carapetis, New normal ranges of antistreptolysin $\mathrm{O}$ and antideoxyribonuclease B titres for Australian children. J. Paediatr. Child Health. 41, 583-586 (2005).

20. S. Erdem, F. Demir, M. Ayana, O. Canan, Y. K. Okuducu, A. Arslan, O. Kucukosmanoglu, N. Özbarlas, Acute rheumatic fever in south-east of Turkey: clinical features and epidemiological evaluation of the patients over the last 25 years. Cardiol. Young. 30, 10861094 (2020). 
21. V. Trushin, M. Englender, Clinical value of antistreptolysin O levels in adult patients with tonsillitis: report I. Eur. Arch. Otorhinolaryngol. 274, 2035-2039 (2017).

22. M. E. Borschmann, R. G. Berkowitz, One-off Streptococcal Serologic Testing in Young Children with Recurrent Tonsillitis. Ann. Otol. Rhinol. Laryngol. 115, 357-360 (2006).

23. R. Ozdemir, C. Karadeniz, O. Doksoz, M. Celegen, Y. Yozgat, B. Guven, T. Meşe, N. Unal, Are Mean Platelet Volume and Platelet Distribution Width Useful Parameters in Children With Acute Rheumatic Carditis? Pediatr. Cardiol. 35, 53-56 (2014).

24. N. Saini, D. Kumar, S. Swarnim, D. Bhatt, S. Kishore, Comparison of antistreptolysin O and anti-deoxyribonucleic B titers in healthy children to those with acute pharyngitis, acute rheumatic fever, and rheumatic heart disease aged 5-15 years. Ann. Pediatr. Cardiol. 12, 195-200 (2019).

25. S. Sethi, K. Kaushik, K. Mohandas, C. Sengupta, S. Singh, M. Sharma, Anti-streptolysin O titers in normal healthy children of 5-15 years. Indian Pediatr. 40, 1068-1071 (2003).

26. A. Abdou, M. Zannoun, M. El Ghanam, Assessment of Anti-Streptolysin-O Titre in Healthy School Age Children in Alexandria- governorate. Int. J. Med. Arts. 0, 0-0 (2020). 